\title{
Evolutionary Neural Network based on Quantum Elephant Herding Algorithm for Modulation Recognition in I mpulse Noise
}

\author{
Hongyuan Gao*, Shihao Wang, Yumeng Su, Helin Sun, Zhiwei Zhang \\ College of Information and Communication Engineering, Harbin Engineering University \\ Harbin, 150001, China \\ [e-mail: gaohongyuan@hrbeu.edu.cn, shihao_wang2020@163.com, suyumeng1994@126.com \\ 2016080213@hrbeu.edu.cn,zhangzhiwei12138@126.com] \\ *Corresponding author: Hongyuan Gao
}

Received April 25, 2021; revised June 5, 2021; accepted June 14, 2021;

published July 31, 2021

\begin{abstract}
In this paper, we proposed a novel modulation recognition method based on quantum elephant herding algorithm (QEHA) evolving neural network under impulse noise environment. We use the adaptive weight myriad filter to preprocess the received digital modulation signals which passing through the impulsive noise channel, and then the instantaneous characteristics and high order cumulant features of digital modulation signals are extracted as classification feature set, finally, the BP neural network (BPNN) model as a classifier for automatic digital modulation recognition. Besides, based on the elephant herding optimization (EHO) algorithm and quantum computing mechanism, we design a quantum elephant herding algorithm (QEHA) to optimize the initial thresholds and weights of the BPNN, which solves the problem that traditional BPNN is easy into local minimum values and poor robustness. The experimental results prove that the adaptive weight myriad filter we used can remove the impulsive noise effectively, and the proposed QEHA-BPNN classifier has better recognition performance than other conventional pattern recognition classifiers. Compared with other global optimization algorithms, the QEHA designed in this paper has a faster convergence speed and higher convergence accuracy. Furthermore, the effect of symbol shape has been considered, which can satisfy the need for engineering.
\end{abstract}

Keywords: impulsive noise, adaptive weight myriad filter, instantaneous characteristics, high order cumulants, quantum elephant herding algorithm, BP neural network. 


\section{Introduction}

The purpose of communication is to transmit information through the channel and to achieve better data transmission, different modulation methods are generally used to modulate the transmission signal in the communication system. Automatic modulation recognition as the intermediate processing of signal reconnaissance and signal demodulation is a crucial technology in the communications domain. The modulation method of identifying wireless communication signals is the basic technology in the fields of electronic countermeasures, electronic reconnaissance, non-cooperative communication, and wireless management. This technology has very wide application and significant value in the civil or military fields.

At present, common modulation signal recognition mainly includes two methods. One is the test method of maximum likelihood hypothesis [1], other is the statistical pattern recognition method according to modulation signals feature extraction [2]. In theory, the performance of the test method of the maximum likelihood hypothesis is optimal. However, the computational complexity of this method is very high. When received prior knowledge is insufficient, it will cause great difficulties to modulation recognition, and this method has high requirements for the establishment of the likelihood function.

The statistical pattern recognition method according to modulation signals feature extraction is another classic method of communication signal modulation recognition. It mainly observes the difference among the characteristic parameters corresponding to different types of modulation signals and selects the classification criteria that meet the requirements to make a decision. And this method is mainly composed of three parts: signal preprocessing, feature extraction, and design classifier. The current commonly used modulation signal feature extraction method includes instantaneous amplitude, frequency, and phase [3], high-order cumulants[4], wavelet-based features[5], and so on. And then, the decision tree (DR) [6], support vector machine (SVM) [7], neural network [8], and other classifiers are trained according to the extracted feature parameters to realize the recognition of modulation signals. The statistical pattern recognition method will find a system that can automatically select the parameters for the modulation signals through a large enough feature training set. Moreover, neural networks and other pattern recognition methods consider all the features at the same time, so the detection speed is fast, the time sequence of the feature will not affect the correct detection probability, as well.

It can be seen from the above literature that most modulation recognition methods are in a Gaussian noise environment. However, the electromagnetic environment faced by wireless communication is very complex, including various interferences and noises, and many of these signals and noises involved are non-Gaussian. Compared with Gaussian noise, one common feature of these noises and signals is they have significant impulsive characteristics and are often called impulsive noise, which can be simulation by alpha-stable distribution [9]. Recently, some scholars have studied modulation recognition in impulsive noise environment, Câmara et al. [10] analyzed the characteristics of the cyclic correntropy function and fractional lower-order cyclic autocorrelation function in impulsive noise environment and designed a robust modulation recognition structure based on these two kinds of cyclic descriptors. X. Tian et al. [11] analyzed fractional low-order Choi Williams distribution transform and found it has better time-frequency performance in the impulsive noise environment, so the author combines convolutional neural network (CNN) and time-frequency distribution of modulation signal to realized modulation recognition in impulsive noise environment. C.C. Wang et al. 
[12] used fractional lower order fast independent component analysis to remove the impulsive noise from the received signal and adopted local mean decomposition to overcome the modal aliasing problem, then extracted instantaneous characteristics of the signal, finally, the DR method is utilized for modulation recognition.

The main method of modulation recognition in impulsive noise environment is to extract fractional low-order cyclic statistical features. This method has a good suppression effect on impulsive noise. However, this method has higher computational complexity, fewer types of features that can be extracted, and poor recognition performance under low mixed signal-to-noise ratio (MSNR). Therefore, this paper uses an adaptive weighted myriad filter [13] to preprocess the input signal to suppress impulsive noise, then extracts a variety of instantaneous characteristics and high order cumulant of modulation signals as classification features, and finally trains BPNN as a classifier to recognize BASK, QASK, BFSK, QFSK, BPSK, QPSK, OQAM and MSK under the impulsive noise environment. But initial thresholds and weights of the BPNN have a great influence on the recognition results, which leads to poor robustness and easily trapped into local minimum value. Hence, this paper designs a QEHA to search for optimal initial thresholds and weights. The simulation results in this paper show that the proposed method not only can identify a variety of digital modulation signals under the impulsive noise, but also has better suppression performance of impulsive noise and higher recognition accuracy, and the impact of symbol shaping [14] on the signal is considered, so it can meet the engineering needs better. The main contributions in this paper are summarized in three parts as follows.

- The adaptive weighted myriad filter is used to preprocess the received modulation signals which passing through the impulsive noise channel. The simulation shows that it has a good suppression effect on the impulsive noise so that the instantaneous characteristics and high order cumulant can be extracted from the preprocessed modulation signals, which makes the classification feature set more diverse and can be applied this feature set for more kinds of modulation signals recognition.

- We design a QEHA based on the EHO algorithm and quantum computing mechanism. We use the quantum rotation gate and quantum not gate to evolve the quantum state of the quantum elephant. In the process of evolution, past historical information of individuals is effectively used, and the global convergence ability of the algorithm is enhanced. Compared with some previous optimization algorithms, it has a faster convergence speed and higher convergence accuracy. And it can find the optimal parameter of BPNN.

- We use the designed QEHA in this paper to optimize the initial thresholds and weights of the BPNN. The experiment result show this method obtains the optimal accuracy under the low MSNR in a modulation recognition system and overcomes the shortcomings of BPNN which is easily trapped in local minimum values and poor robustness. And compared with other pattern recognition methods, the recognition of QEHA-BPNN is significantly improved.

The rest of this paper is systemized in the following: received signal preprocessing is shown in Section 2. In Section 3, the instantaneous characteristics and high order cumulant of modulation signals are extracted. In Section 4, BPNN based on QEHA for modulation is proposed. Experimental results are presented in Section 5 and the conclusion in Section 6. 


\section{Received Signal Preprocessing}

As shown in Fig. 1, the modulation type identified in this paper contains a variety of different modulation signals. These modulated signals pass through the symbol shaping filter at the transmitter, then arrive at the receiver through the impulsive noise channel, and the preprocessed signal is finally obtained via the adaptive weighted myriad filter.

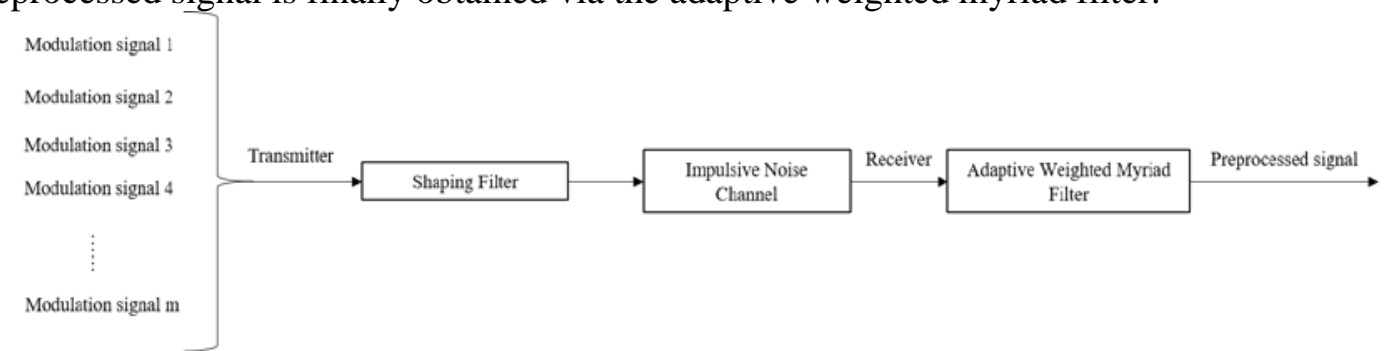

Fig. 1. Received signal preprocessing.

\subsection{Digital Modulation Model}

Digital signal modulation is to change and control some parameters of the carrier by modulating the signal. These parameters will change with some rules of the modulated signal itself. The modulated signal can carry the same information as the original to ensure that the information is safe, effective, and lossless in the process of channel transmission. Different modulation types can be obtained by changing the frequency, amplitude, and phase characteristics of the carrier. The commonly used modulation types mathematical models are shown below.

Amplitude-shift keying (ASK) mainly transmits signals by changing the amplitude of the carrier, and its expression is

$$
S_{\text {MASK }}(t)=\left[\sum_{n} \alpha_{n} g\left(t-n T_{s}\right)\right] \cos \left(2 \pi f_{c} t+\varphi\right)
$$

where $\alpha_{n}$ denotes the level value of the $n$-th transmission symbol and $\alpha_{n} \in\{0,1, \ldots, M-1\}, M$ is the modulation number, $f_{c}$ is the carrier frequency, $g(\cdot)$ is the rectangular pulse with amplitude value of 1 and duration of $T_{s}, T_{s}$ is the symbol period, and $\varphi$ is the initial phase of the carrier.

Frequency-shift keying (FSK) mainly transmits signals by changing the frequency of the carrier, which can be expressed as

$$
S_{\text {MFSK }}(t)=\left[\sum_{n} g\left(t-n T_{s}\right)\right] \cos \left(2 \pi\left(f_{c}+b_{n} \Delta f\right) t+\varphi\right)
$$

where $b_{n}$ represents the frequency offset multiple of the $n$-th transmission symbol and $b_{n} \in\{0,1, \ldots, M-1\}, \Delta f$ is the frequency offset of the carrier wave and is usually taken as $\frac{1}{2 T_{s}}$, and the initial phase $\varphi$ of MFSK modulation is generally taken as 0 .

Phase-shift keying (PSK) mainly transmits signals by changing the phase of the carrier, and its expression is

$$
S_{\text {MPSK }}(t)=\left[\sum_{n} g\left(t-n T_{s}\right)\right] \cos \left(2 \pi f_{c} t+\varphi_{n}\right)
$$

where $\varphi_{n}$ represents the phase corresponding to the $n$-th symbol, $\varphi_{n}=2 \pi(n-1) / M$ and $n=1,2, \ldots, M$. 
Quadrature amplitude modulation (QAM) mainly transmits signals by changing the amplitude and phase simultaneously of the carrier, and its expression is

$$
S_{\text {мемм }}(t)=\left[\sum_{n} a_{n} g\left(t-n T_{s}\right)\right] \cos \left(2 \pi f_{c} t+\varphi_{n}\right)
$$

where $a_{n}$ represents the amplitude of the $n$-th transmission symbol, $\varphi_{n}$ denotes the initial phase of the $n$-th transmission symbol.

Minimum shift keying (MSK) mainly transmits signals by changing the frequency of the carrier which is a special kind of continuous phase frequency shift keying, and its expression is

$$
S_{\text {MSK }}(t)=\cos \left(2 \pi f_{c} t+\frac{\pi b_{n} t}{2 T_{s}}+\varphi_{n}\right)
$$

where $b_{n}$ represents the $n$-th transmitted symbol, and $\varphi_{n}$ represents the initial phase of the $n$-th transmission symbol.

\subsection{Shaping Filter}

The digital baseband signal is a rectangular wave without symbol shaping, which is infinitely extended in the frequency domain. In a signal with limited bandwidth, it will cause waveform distortion at the receiver. In order to eliminate waveform distortion and ensure no new inter symbol interference, in practice, the transmitter will adopt a raised cosine roll-off function to shape the digital baseband signal. The expression of the raised cosine roll-off function is:

$$
q(t)=\frac{\sin (\pi t / T)}{(\pi t / T)} \frac{\cos (\delta \pi t / T)}{\left[1-\left(4 \delta^{2} t^{2} / T^{2}\right)\right]}
$$

where $t$ is the sampling time, $T$ is the symbol period, and $-3 T<t<3 T, \delta$ is the roll-off factor.

\subsection{Impulsive Noise Model}

Impulsive noise refers to some noise that has a significant spike pulse waveform and heavy-tailed behavior in the practical wireless communication system. Generally, alpha-stable distribution is used to establish the simulation model of impulsive noise, and which is defined by the following characteristic function:

$$
\varphi(t)=\left\{\begin{array}{l}
\exp \left\{j \mu t-\gamma|t|^{\alpha}\left[1+j \beta \operatorname{sgn}(t) \tan \left(\frac{\alpha \pi}{2}\right)\right]\right\}, \text { if } \alpha \neq 1 \\
\exp \left\{j \mu t-\gamma|t|^{\alpha}\left[1+j \beta \operatorname{sgn}(t) \frac{2}{\pi} \log |t|\right]\right\}, \text { if } \alpha=1
\end{array}\right.
$$

where $\operatorname{sgn}(t)=\left\{\begin{array}{l}1, t>0 \\ 0, t=0 \\ -1, t<0\end{array}, 0<\alpha \leq 2\right.$ is the characteristic exponent, which is related to the impact of alpha-stable distribution, $-1<\beta \leq 1$ is the symmetrical parameter, which indicates the symmetry degree of alpha-stable distribution, $\gamma \geq 0$ is the dispersion coefficient, which represents the dispersion degree of alpha-stable noise, $\mu$ is displacement parameter, $\mu$ is median value when $0<\alpha \leq 1$, and $\mu$ is mean value when $1<\alpha \leq 2$.

Because the stable distribution has no finite second-order moment, so MSNR is usually used to describe SNR in the impulsive noise environment

$$
\operatorname{MSNR}_{d B}=10 \log 10\left(\sigma_{s}^{2} / \gamma\right)
$$

where $\sigma_{s}^{2}$ is signal variance. 


\subsection{Adaptive Weighted Myriad Filters}

There are mainly two methods for modulation recognition in an impulsive noise environment, one is to extract the features of fractional lower order statistics which are also effective in the non-Gaussian environment [9-11], and the other is to remove impulsive noise [12]. In this paper, an adaptive weight myriad filter [13] is used, which can effectively remove impulsive noise and extract information with less distortion.

The sample myriad is denotes as the maximum likelihood estimation of the location parameters of the data, and these data obey the Cauchy distribution. Define the input vector $\boldsymbol{x}=\left[x_{1}, x_{2}, \ldots, x_{N}\right]^{\mathrm{T}}$ and weight vector $\boldsymbol{\omega}=\left[\omega_{1}, \omega_{2}, \ldots, \omega_{N}\right]^{\mathrm{T}}$, for a given linearity parameter $K$, it is assumed that the random variable obeys the Cauchy distribution of the position parameter $\theta$ and the scale parameter $\left\{S_{i}\right\}_{i=1}^{N}$, where

$$
S_{i}=\frac{K}{\sqrt{\omega_{i}}}>0
$$

Then the weighted myriad can be denotes as

$$
\hat{\theta}(\boldsymbol{\omega}, \boldsymbol{x})=\arg \min _{\theta} \prod_{i=1}^{N}\left[1+\left(\frac{x_{i}-\theta}{S_{i}}\right)^{2}\right]=\arg \min _{\theta} \prod_{i=1}^{N}\left[1+\omega_{i}\left(\frac{x_{i}-\theta}{K}\right)^{2}\right]
$$

Since $\log (\cdot)$ is a strictly increasing function, weight myriad can also be expressed as

$$
\hat{\theta}(\boldsymbol{\omega}, \boldsymbol{x})=\arg \min _{\theta} \sum_{i=1}^{N} \log \left[1+\left(\frac{x_{i}-\theta}{S_{i}}\right)^{2}\right]
$$

There are two important parameters in the weighted myriad filter, the linearity parameter $K$ and the weights $\boldsymbol{\omega}$. The linearity parameter $K>0$ is related to the characteristic exponent $\alpha$ and dispersion coefficient $\gamma$ of the impulsive noise. The empirical formula for $K$ is as follow

$$
K=\gamma \sqrt{\frac{\alpha}{2-\alpha}}
$$

The weights $\left\{\omega_{i} \geq 0\right\}_{i=1}^{N}$ is calculated by the adaptive weight estimation method based on the minimum root mean square. If the input of weighted myriad filter is $\left\{x_{i}\right\}_{i=1}^{N}$, and the output is $y(\boldsymbol{\omega}, \boldsymbol{x})$, the expected output value is $d$, the adaptive algorithm for updating the filter weight $\omega_{i}$ is as follow

$$
\omega_{i}(n+1)=P\left\{\omega_{i}(n)+\lambda \operatorname{sgn}(l-d) \times \frac{\left(y-x_{i}\right)}{\left[1+\frac{\omega_{i}}{K^{2}}\left(y-x_{i}\right)^{2}\right]^{2}}(n)\right\}
$$

where $\lambda$ is step size and the function $P(\cdot)$ is defined as :

$$
P(u)=\left\{\begin{array}{l}
u, u>0 \\
0, u \leq 0
\end{array}\right.
$$




\section{Feature Set}

In this paper, the statistical pattern recognition method according to modulation signals feature extraction is adopted. The classification criteria that meet the requirements are selected to make a decision by observing the difference among the corresponding feature parameters of different types of modulation signals. Constructing feature sets that can distinguish different modulation signals is an important part of this method. This paper mainly extracts instantaneous characteristics and high-order cumulant features of modulation signals to construct a feature set.

\subsection{Instantaneous Feature}

The frequency, amplitude, and phase of the communication signals contain modulation information. The instantaneous frequency, amplitude, and phase of the signal can be extracted from the received modulation signal, and some characteristic parameters can be constructed from these instantaneous parameters, which are called instantaneous features. The extracted instantaneous feature technique for modulation recognition as following.

1) Average value of instantaneous amplitude envelope

$$
m_{\mathrm{a}}=\frac{1}{N} \sum_{i=1}^{N} A(i)
$$

where $A(i)$ is the instantaneous amplitude of communication signal, and $N$ denotes the number of samples. The average value of instantaneous amplitude envelope reflects the change characteristics of communication signal envelope.

2) The maximum value of the instantaneous amplitude power spectral density of the normalized center

$$
r_{\max }=\max \left|\operatorname{DFT}\left(A_{\mathrm{cn}}(i)\right)\right|^{2} / N
$$

where $A_{c n}(i)$ is the value of instantaneous amplitude of the normalized center, and it is defined by

$$
A_{c n}(i)=A_{n}(i)-1
$$

where $A_{n}(i)=\frac{A(i)}{m_{a}}$. The maximum value of the instantaneous amplitude power spectral density of the normalized center $r_{\max }$ represents the change of instantaneous amplitude of signal, which can distinguish constant envelope modulation and non-constant envelope modulation.

3) Standard deviation of the instantaneous amplitude of the normalized center of the non-weak signal segment

$$
\sigma_{\mathrm{da}}=\sqrt{\frac{1}{W}\left[\sum_{A_{\mathrm{n}}(i)>a_{t}} A_{\mathrm{cn}}^{2}(i)\right]-\left[\frac{1}{W} \sum_{A_{\mathrm{n}}(i)>a_{t}} A_{\mathrm{cn}}(i)\right]^{2}}
$$

where $W$ is the number of non-weak signal values in all $N$ samples, and non-weak signal refers to a signal which signal amplitude $A_{n}(i)$ is greater than the amplitude decision threshold level $a_{t}$.

4) Standard deviation of the absolute value of the instantaneous amplitude of the normalized center

$$
\sigma_{\mathrm{aa}}=\sqrt{\frac{1}{N}\left[\sum_{i=1}^{N} A_{\mathrm{cn}}^{2}(i)\right]-\left[\frac{1}{N} \sum_{i=1}^{N} \mid A_{\mathrm{cn}}(i)\right]^{2}}
$$

5) Kurtosis of the normalized-centered instantaneous amplitude 


$$
\mu_{42}^{a}=\mathrm{E}\left[A_{\mathrm{cn}}^{4}(i)\right] / \mathrm{E}\left[A_{\mathrm{cn}}^{2}(i)\right]^{2}
$$

6) Standard deviation of the centered non-linear components instantaneous phase in non-weak signal segment

$$
\sigma_{\mathrm{dp}}=\sqrt{\frac{1}{W}\left(\sum_{A_{n}(i)>a_{t}} \phi_{\mathrm{NL}}^{2}(i)\right)-\left(\frac{1}{W} \sum_{A_{\mathrm{n}}(i)>a_{t}} \phi_{\mathrm{NL}}(i)\right)^{2}}
$$

where $\phi_{\mathrm{NL}}(i)$ is the normalized-centered non-linear component of the instantaneous phase, and it is defined as follows

where $\phi_{0}=\frac{1}{N} \sum_{i=1}^{N} \phi(i)$.

$$
\phi_{\mathrm{NL}}(i)=\phi(i)-\phi_{0}
$$

7) Standard deviation of absolute value of intermediate nonlinear component of instantaneous phase in non weak signal segment

$$
\sigma_{\mathrm{ap}}=\sqrt{\frac{1}{W}\left(\sum_{A_{n}(i)>a_{t}} \phi_{\mathrm{NL}}^{2}(i)\right)-\left(\frac{1}{W} \sum_{A_{n}(i)>a_{t}}\left|\phi_{\mathrm{NL}}(i)\right|\right)^{2}}
$$

8) Variance of normalized instantaneous frequency

$$
\delta_{\mathrm{f}}^{2}=\frac{1}{N} \sum_{i=1}^{N}\left[f(i)-\frac{1}{N} \sum_{i=1}^{N} f(i)\right]^{2}
$$

where $f(i)$ is instantaneous frequency.

9) Standard deviation of absolute value of instantaneous frequency centered on normalization in non weak signal segment

where

$$
\sigma_{\mathrm{af}}=\sqrt{\frac{1}{W}\left(\sum_{A_{\mathrm{n}}(i)>a_{t}} f_{\mathrm{N}}^{2}(i)\right)-\left(\frac{1}{W} \sum_{A_{\mathrm{n}}(i)>a_{t}}\left|f_{\mathrm{N}}(i)\right|\right)^{2}}
$$

$$
\begin{gathered}
f_{\mathrm{N}}(i)=f_{\mathrm{c}}(i) / r_{\mathrm{b}} \\
f_{\mathrm{c}}(i)=f(i)-\frac{1}{N_{\mathrm{s}}} \sum_{i=1}^{N_{\mathrm{s}}} f(i)
\end{gathered}
$$

$r_{\mathrm{b}}$ is the symbol rates.

10) Kurtosis of the normalized-centered instantaneous frequency

$$
\mu_{42}^{f}=\mathrm{E}\left[f_{\mathrm{cn}}^{4}(i)\right] / \mathrm{E}\left[f_{\mathrm{cn}}^{2}(i)\right]^{2}
$$

where, $f_{c n}(i)=\frac{f(i)}{m_{f}}-1, m_{f}=\frac{1}{N} \sum_{i=1}^{N} f(i)$.

\subsection{High Order Cumulants}

High order cumulant features are widely used in the field of digital modulation recognition. For a zero-mean complex stationary process $X(t)$, the $p_{\text {-order mixing moment is defined as }}$

$$
M_{p q}=E\left\{[X(t)]^{p-q}\left[X^{*}(t)\right]^{q}\right\}
$$

Where '*' denotes conjugate. Then the expression of each order cumulant is

1) Second-order cumulant

2) Forth-order cumulant

$$
\begin{aligned}
& C_{20}=M_{20}=E\left\{[X(t)]^{2}\right\} \\
& C_{21}=M_{21}=E[|X(t)|]^{2}
\end{aligned}
$$




$$
\begin{gathered}
C_{40}=M_{40}-3\left(M_{20}\right)^{2} \\
C_{41}=M_{41}-3 M_{20} M_{21} \\
C_{42}=M_{42}-\left|M_{20}\right|^{2}-2\left(M_{21}\right)^{2}
\end{gathered}
$$

3) Sixth-order cumulant

$$
\begin{gathered}
C_{60}=M_{60}-15 M_{20} M_{40}+30\left(M_{20}\right)^{3} \\
C_{63}=M_{63}-9 M_{42} M_{21}+9\left|M_{20}\right|^{2} M_{21}+12\left(M_{21}\right)^{3}
\end{gathered}
$$

The selected four identification parameters are set as $f_{s 1}=\frac{\left|C_{40}\right|}{\left|C_{42}\right|}, f_{s 2}=\frac{\left|C_{41}\right|}{\left|C_{42}\right|}, f_{s 3}=\frac{\left|C_{60}\right|}{\left|C_{21}\right|^{3}}$, and $f_{s 4}=\frac{\left|C_{63}\right|}{\left|C_{21}\right|^{3}}$

\section{BPNN Based on QEHA for Modulation Recognition}

Classifier design is an extremely important part of modulation signal recognition. The previous signal preprocessing and feature set construction determines the superior limit, and a good classifier can make the recognition result approach the superior limit. With the continuous development of machine learning, the BPNN as a classifier is widely used in the domain of pattern recognition, which has strong self-learning and self-adaptive capabilities and can deal with complex nonlinear problems. This section introduces the application of a backpropagation neural network based on the quantum elephant herding algorithm (QEHA-BPNN) in wireless communication modulation recognition.

\subsection{Quantum Elephant Herding Algorithm (QEHA)}

The EHO algorithm is a global random search algorithm proposed by Wang et al. [15] which is based on the study of the herding behavior of the elephant group. EHO algorithm has been widely used in optimizing machine learning. In the literature [16], use EHO algorithm to optimize neural network and applied to cancer prediction, the simulation shows that the modulation recognition accuracy is significantly improved. However, the EHO algorithm does not change the information of the elephant individual, and the search is affected by the unreasonable convergence of the update operator [17]. For this reason, this paper proposed a quantum elephant herding algorithm (QEHA), which uses signal chain coding, quantum rotation gate, and quantum not gate to evolve its quantum position.

Quantum computing is a research field including quantum mechanical computers and quantum algorithms. The combination of quantum mechanics and other classical optimization methods mainly focuses on two aspects: one is design new quantum algorithms in classic computers; the other is to introduce the quantum idea into classical optimization algorithms and improve traditional algorithms to obtain a better performance [18]. QEHA is based on the second consideration.

Based on quantum state evolution of elephant herding behavior, a novel QEHA is proposed. In a $D$-dimensional problem, at the beginning of QEHA, the quantum position of $P$ elephants are randomly generated within the domain of the qubit, the quantum position of a quantum elephant is represented by a string of qubits. And a qubit can be represented by a complex pair, which can be express as $(\alpha, \beta)^{T}$, where $|\alpha|^{2}+|\beta|^{2}=1$. The quantum position of $i$-th $(i=1,2, \ldots, P)$ quantum elephant of the $t$-th iteration can be defined as 


$$
\boldsymbol{w}_{i}^{t}=\left[\begin{array}{l}
\alpha_{i, 1}^{t}, \alpha_{i, 2}^{t}, \ldots, \alpha_{i, D}^{t} \\
\beta_{i, 1}^{t}, \beta_{i, 2}^{t}, \ldots, \beta_{i, D}^{t}
\end{array}\right]
$$

where $\left|\alpha_{i, j}^{t}\right|^{2}+\left|\beta_{i, j}^{t}\right|^{2}=1$, in which $j=1,2, \ldots, D, \alpha_{i, j}^{t}$ and $\beta_{i, j}^{t}$ are defined as $0 \leq \alpha_{i, j}^{t} \leq 1$ and $0 \leq \beta_{i, j}^{t} \leq 1$ respectively. Because of $\beta_{i, j}^{t}=\sqrt{1-\left(\alpha_{i, j}^{t}\right)^{2}}$, the quantum position of the $i$-th quantum elephant can be defines as

$$
\boldsymbol{w}_{i}^{t}=\left[\alpha_{i, 1}^{t}, \alpha_{i, 2}^{t}, \ldots, \alpha_{i, D}^{t}\right]=\left[w_{i, 1}^{t}, w_{i, 2}^{t}, \ldots, w_{i, D}^{t}\right]
$$

where $0 \leq w_{i, j}^{t} \leq 1$, and $w_{i, j}^{t}$ denotes the $j$-th quantum bit of the quantum elephant $i$. The mapping position of the $i$-th quantum elephant is obtained by

$$
\hat{\hat{w}}_{i, j}^{t}=\hat{\hat{w}}_{i, j}^{\min }+\left(\hat{\hat{w}}_{i, j}^{\max }-\hat{\hat{w}}_{i, j}^{\min }\right) \cdot \stackrel{\underline{w}}{t}_{i, j}^{t}
$$

where $\hat{w}_{i, j}^{t}$ represent the $j$-th position of the $i$-th quantum elephant in $t$-th iterations, $\hat{w}_{i, j}^{\max }$ and $\hat{w}_{i, j}^{\text {hin }}$ is the upper bound and lower bound of the searching range respectively. The position of each quantum elephant corresponds to a feasible solution of the optimization problem.

In an evolution, all the elephants are arranged in ascending order according to their fitness values $f\left(\hat{\boldsymbol{w}}_{i}^{t}\right)$, where $f(\cdot)$ is fitness function. Then all the elephants are divided into $M$ clans, and each clan contains $n$ elephants $(P=M \times n)$. In the process of division, the first elephant entered the first clan, the second elephant entered the second clan, ... , the $M$-th elephant entered the $M$-th clan, then the $M+1$-th elephant into the first clan, and so on, until all the elephants are divided. The elephant with most suitable in each clan is the matriarch, which will affect the quantum position update of other elephants in the clan updating operator, while the elephant which has worst fitness value will perform the separation operator, so that the search has a better distribution in each generation.

Elephants in each clan live under the leadership of matriarch, so the next generation quantum position of each elephant is influenced by the matriarch and implement clan updating operator. Therefore the $j$-th qubit of the $i$-th elephant quantum position in the $m$-th clan is updated as follows

$$
\begin{gathered}
\theta_{i, j}^{t+1}(m)=r_{1}^{t} \tau\left[w_{i, j}^{t}(m)-w_{b, j}^{t}(m)\right]+r_{2}^{t}(1-\tau)\left[w_{i, j}^{t}(m)-w_{c, j}^{t}(m)\right] \\
w_{i, j}^{t+1}(m)=\left\{\begin{array}{l}
\sqrt{1-\left(w_{i, j}^{t}(m)\right)^{2}}, \quad \text { if } \theta_{i, j}^{t+1}(m)=0 \text { and } r_{3}^{t}<c_{1} ; \\
\operatorname{abs}\left(w_{i, j}^{t}(m) \cdot \cos \theta_{i, j}^{t+1}(m)+\sqrt{1-\left(w_{i, j}^{t}(m)\right)^{2}} \cdot \sin \theta_{i, j}^{t+1}(m)\right), \quad \text { else. }
\end{array}\right.
\end{gathered}
$$

where $i=1,2, \ldots, n ; j=1,2, \ldots, D ; m=1,2, \ldots, M ; r_{1}^{t}, r_{2}^{t}$ and $r_{3}^{t}$ are random number in the range of 0 and $1 ; t$ represents the number of iterations; $c_{1} \leq 1 / D$ is the mutation probability; $\tau \in[0,1]$ is the influencing factor, which determines the influence of the matriarch on the position of the next generation; $\boldsymbol{w}_{b}^{t}(m)=\left[w_{b, 1}^{t}(m), w_{b, 2}^{t}(m), \ldots, w_{b, D}^{t}(m)\right]$ is the quantum position of the elephant with the best fitness value in the clan $m$, that is, the quantum position of the matriarch; $\boldsymbol{w}_{c}^{t}(m)=\left[w_{c, 1}^{t}(m), w_{c, 2}^{t}(m), \ldots, w_{c, D}^{t}(m)\right]$ is the center position of the clan $m$, which can be expressed as the following formula 


$$
w_{c, j}^{t}(m)=\frac{1}{n} \times \sum_{i=1}^{n} w_{i, j}^{t}(m)
$$

When the male elephant reaches puberty, it will leave their clan and live alone away from the elephant population. When solving optimization problems, this behavior can be modeled as separation operator. The elephant with the worst fitness value as an adult elephant will implement the separating operator as follows

$$
\begin{gathered}
\bar{\theta}_{j}^{t+1}(m)=\bar{r}_{1}^{t} s_{j}^{t}\left[w_{d, j}^{t}(m)-w_{g, j}^{t}\right]+\bar{r}_{2}^{t} \bar{s}_{j}^{t}\left[w_{d, j}^{t}(m)-w_{c, j}^{t}(m)\right] \\
\bar{w}_{j}^{t+1}(m)=\left\{\begin{array}{l}
\sqrt{1-\left(w_{d, j}^{t}(m)\right)^{2}}, \quad \text { if } \bar{\theta}_{j}^{t+1}(m)=0 \text { and } \bar{r}_{3}^{t}<c_{2} ; \\
\operatorname{abs}\left(w_{d, j}^{t}(m) \cdot \cos \bar{\theta}_{j}^{t+1}(m)+\sqrt{1-\left(w_{d, j}^{t}(m)\right)^{2}} \cdot \sin \bar{\theta}_{j}^{t+1}(m)\right), \quad \text { else. }
\end{array}\right.
\end{gathered}
$$

where $\bar{r}_{1}^{t}, \quad \bar{r}_{2}^{t}$ and $\bar{r}_{3}^{t}$ are random number in the range of 0 and 1 ; $\boldsymbol{w}_{d}^{t}(m)=\left[w_{d, 1}^{t}(m), w_{d, 2}^{t}(m), \ldots, w_{d, D}^{t}(m)\right]$ is the worst elephant individual in clan $m$; $\boldsymbol{w}_{g}^{t}=\left[w_{g, 1}^{t}, w_{g, 2}^{t}, \ldots, w_{g, D}^{t}\right]$ is the elephant quantum position with the best fitness value in the entire population; The chaotic variables $s_{j}^{t}$ and $\bar{s}_{j}^{t}$ satisfy the chaotic equation $s_{j}^{t}=4 s_{j}^{t-1}\left(1-s_{j}^{t-1}\right)$ and $\bar{s}_{j}^{t}=4 \bar{s}_{j}^{t-1}\left(1-\bar{s}_{j}^{t-1}\right)$ respectively; $c_{2} \leq 1 / D$ is the mutation probability.

\subsection{BPNN Optimized by QEHA}

Because the neural network has powerful pattern recognition capabilities, each node in the neural network automatically and adaptively selects the threshold of the characteristic parameter and considers all the characteristic parameters simultaneously so that the modulation recognition accuracy is not restricted by the order of using characteristic parameters. This paper uses BPNN with three-layer network structure as the classifier. The number of nodes in the input layer and output layer is determined by the number of input features types and the number of output modulation signal types, respectively. Gave the formula to determine the number of hidden layer nodes

$$
\hat{h}=\sqrt{\hat{m}+\hat{n}}+a
$$

where $\hat{m}$ is the number of input features types; $\hat{n}$ is the number of output modulation signal types; $a \in[1,10]$ is a constant, so the number of BPNN hidden layer nodes is an integer in range $[\sqrt{\hat{m}+\hat{n}}+1, \sqrt{\hat{m}+\hat{n}}+10]$.

In general, the initial thresholds and weights in the BPNN are usually taken as a random number between -1 and 1 , which will affect the training speed, training results, and convergence, resulting in poor robustness of the BPNN. Therefore, obtaining the optimal initial weights and thresholds will greatly enhance the performance of the BPNN. In this paper, the QEHA is utilized to optimize the initial thresholds and weights of the BPNN, which is called QEHA-BPNN.

In order to predict the output of the system, the feature set is utilized to train the BPNN, and the goal of fitness function is the mean absolute error (MAE) between the BPNN output layer output and the expected output. The optimal solution equation can be described as following 


$$
f(\hat{\boldsymbol{w}})=\frac{1}{\hat{n}} \sum_{i=1}^{\hat{n}}\left|\hat{Y}_{i}-O_{i}\right|
$$

where $\hat{w}=\left[\hat{w}_{1}, \hat{w}_{2}, \ldots, \hat{w}_{D}\right]$ is the combined vector of the initial weights and thresholds of the BPNN, so the initial weights is $\hat{w}_{1}=\left[\hat{w}_{1}, \hat{w}_{2}, \ldots, \hat{w}_{d 1}\right]$ and thresholds is $\hat{b}_{1}=\left[\hat{w}_{d 1+1}, \hat{w}_{d 2+2}, \ldots, \hat{w}_{d 2}\right]$ between the input layer and hidden layer, and the initial weights is $\hat{w}_{2}=\left[\hat{w}_{d 2+1}, \hat{w}_{d 2+2}, \ldots, \hat{w}_{d 3}\right]$ and thresholds is $\hat{b}_{2}=\left[\hat{w}_{d 3+1}, \hat{w}_{d 3+2}, \ldots, \hat{w}_{D}\right]$ between the hidden layer and output layer,where, $d 1=\hat{n} \times \hat{h}, d 2=\hat{n} \times \hat{h}+\hat{h}, d 3=\hat{n} \times \hat{h}+\hat{h}+\hat{h} \times \hat{m}$, and $D$ is the total number of nodes in the neural network and which can be described as $D=\hat{n} \times \hat{h}+\hat{h}+\hat{h} \times \hat{m}+\hat{m} ; \hat{\boldsymbol{Y}}=\left[\hat{Y}_{1}, \hat{Y}_{2}, \ldots, \hat{Y}_{\hat{n}}\right]$ is the expected output; $\boldsymbol{O}=\left[O_{1}, O_{2}, \ldots, O_{\hat{n}}\right]$ is the predicted output.

According to the above design and analysis, the flowchart of BPNN based on QEHA for modulation recognition proposed in this paper is shown in Fig. 2, and the implementation process is shown in the following steps.

Step1: Initialize the parameters, including the total number of elephant groups $P$, the number of elephant clans $M$, and the number of elephants in each clan $n, P=M \times n$; the maximum number of iterations Gen; impact factor $\tau$; qubit mutation probability $c_{1}$ and $c_{2}$; Generate the initial quantum position of the elephant in the quantum position domain randomly.

Step2: Use (39) map the quantum position to the position, calculate the fitness value $f\left(\hat{\boldsymbol{w}}_{i}^{t}\right)$ of each elephant use (46), and arrange the elephants in ascending order according to the fitness value. The quantum position of the elephant with the global optimal fitness value is $\boldsymbol{w}_{g}^{t}$.

Step3: Divide all groups into $M$ clans, the quantum positions of elephants with the best fitness and the worst fitness in the m-th clans are recorded as $\boldsymbol{w}_{b}^{t}(m)$ and $\boldsymbol{w}_{d}^{t}(m)$ respectively.

Step4: Use (40), (41), and (42) to perform the clan updating operator to update the elephant quantum position.

Step5: Use (43) and (44) to execute the separation operator to replace the elephant individual with the worst fitness value in the clan.

Step6: Merge the elephants of each clan, use (39) map the quantum position to the position, use (46) to calculate the fitness value $f\left(\hat{\boldsymbol{w}}_{i}^{t+1}\right)$ of each elephant, and arrange the elephants in ascending order of fitness value to determine the elephant position with the global optimal fitness value.

Step7: If the stop condition is not meet, return to step 3, otherwise output the global optimal position $\hat{\boldsymbol{w}}=\left[\hat{w}_{1}, \hat{w}_{2}, \ldots, \hat{w}_{D}\right]$, and terminate the algorithm.

Step8: Training the network with optimal initial thresholds and weights, and then calculate the recognition accuracy of trained BPNN model. 


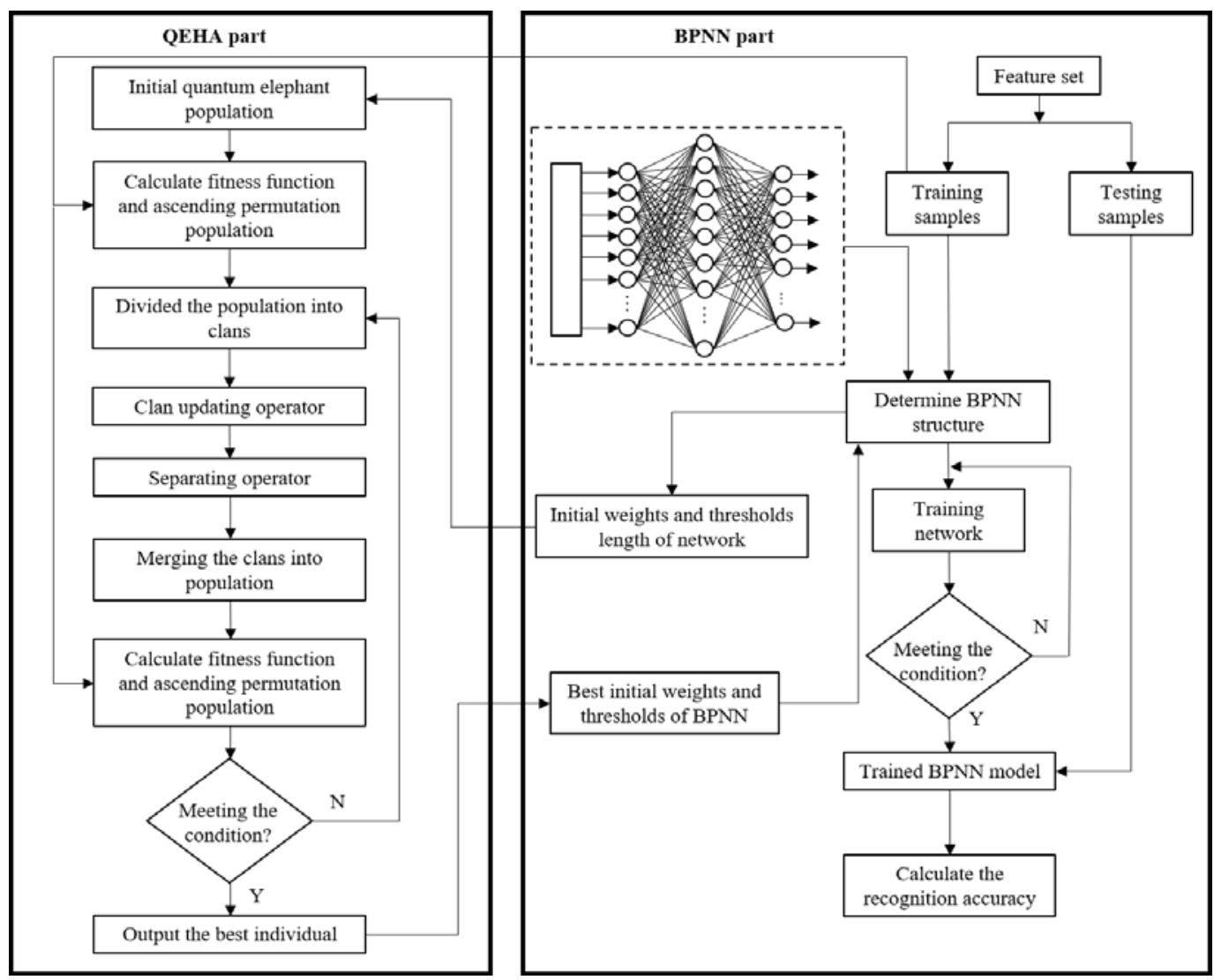

Fig. 2. The flowchart of BPNN based on QEHA for modulation recognition.

\section{Simulation Results}

In this section, we evaluate the overall performance of the proposed BPNN based on QEHA for modulation recognition in impulsive noise environment. The modulation recognition signals include BASK, QASK, BFSK, QFSK, BPSK, QPSK, OQAM, and MSK. We use the MSNR to describe the signal and noise power ratio and given by equation (8), and the simulation parameter of modulation signals and alpha-stable distribution model is presented in Table 1.

Table 1. Simulation parameters of modulation signals and alpha-stable distribution model

\begin{tabular}{ccc}
\hline \hline Symbol & Parameter & Value \\
\hline$\delta$ & Rolloff factor & 0.4 \\
$f_{s}$ & Sampling frequency & $3.264 \mathrm{MHz}$ \\
$f_{c}$ & Carrier frequency & $408 \mathrm{KHz}$ \\
$f_{D}$ & Symbol rate & $38.4 \mathrm{KHz}$ \\
$f_{d}$ & Number of samples per symbol & 85 \\
$N_{s}$ & Number of symbols in each signal & 22 \\
$\beta$ & Symmetrical parameter & 0 \\
$\mu$ & displacement parameter & 0 \\
$\alpha$ & characteristic exponent & {$[0.1,1.9]$} \\
MSNR & mixed signal-to-noise ratio & {$[-10,10]$} \\
\hline \hline
\end{tabular}


The simulation parameters of the QEHA-BPNN is presented in Table 2.

Table 2. Simulation parameters of QEHA-BPNN

\begin{tabular}{ccc}
\hline \hline Symbol & Parameter & Value \\
\hline$\hat{m}$ & Number of input layer nods & 14 \\
$\hat{h}$ & Number of output layer nods & 12 \\
$\hat{n}$ & Number of hidde layer nods & 8 \\
$l r$ & BPNN learning rate & 0.01 \\
goal & BPNN training target error & 0.001 \\
epochs & BPNN maximum number of cycles & 1000 \\
Gen & QEHA maximum number of iteration & 100 \\
$P$ & Population size of the quantum elephant herding & 30 \\
$M$ & Number of the clans & 5 \\
$n$ & Number of quantum elephant in each clans & 6 \\
$\tau$ & Influencing factor & 0.4 \\
\hline \hline
\end{tabular}

\subsection{Performance Comparisons of Adaptive Weighted Myriad Filter}

In this paper, we use an adaptive weighted myriad filter to suppress the impulsive noise. As showing in Fig. 3 representing the preprocessing processes of input digital modulation signal. Fig. 3 shows the preprocessing process of input digital modulation signal BFSK, Fig. 3 (a) shows that the BFSK modulation is used to transmit signals by changing the amplitude of the carrier, Fig. 3 (b) processes the input digital modulation signal BFSK through a shaping filter to eliminate the waveform distortion at the receiving end. Fig. 3 (c) shows the waveform of the output signal through an additive impulsive noise channel with $\alpha=1.5$ and $M S N R=0$. And Fig. 3 (d) shows the waveform of the output signal after being processed by a weighted myriad filter. From Fig. 3 we can see the impulsive noise is removed and the feature of BFSK is retained.
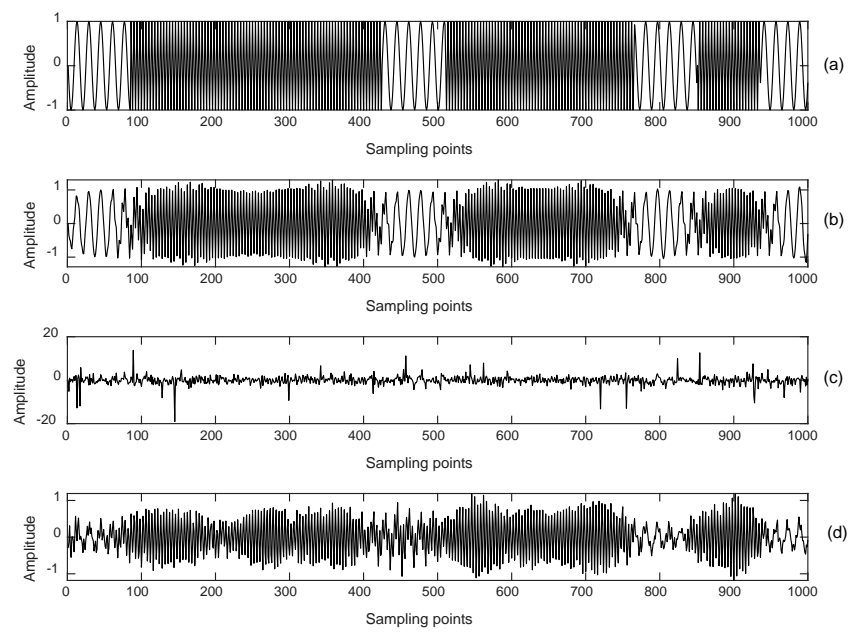

Fig. 3. BFSK signal preprocessing process. 
Fig. 4 shows the modulation recognition accuracy curve after processing by adaptive weighted myriad filter (My-BPNN) and the modulation recognition accuracy curve without the adaptive weighted myriad filters (Not-My-BPNN). The classifier is the traditional BPNN, under the conditions of MSNR $=0 \mathrm{~dB}$ and the characteristic exponent $\alpha$ is 0.1 to 1.9 with interval 0.1. By observing Fig. 4 the simulation shows that the modulation recognition accuracy is greatly increased after using the adaptive weighted myriad filter. And when the characteristic exponent $\alpha=1.0$, symmetrical parameter $\beta=0$ and displacement parameter $\mu=0$, the alpha-stable distribution is Cauchy distribution. Therefore, we can conclude that accuracy of modulation recognition when $\alpha=1.0$ is significantly higher than other cases.

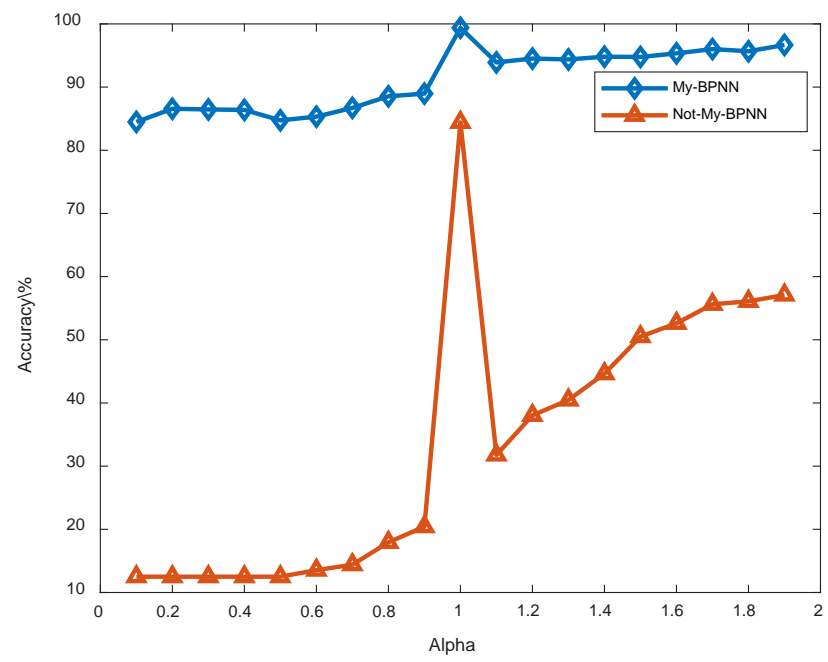

Fig. 4. Overall recognition accuracy of passed adaptive weight Myriad filters and not passed adaptive weight Myriad filters with MSNR $=0 \mathrm{~dB}$ in different characteristics exponent.

\subsection{Performance Comparisons of QEHA-BPNN with Traditional Pattern Recognition Method}

In order to show off the experiment result, Fig. 5 exhibits the confusion matrix calculated by the proposed method QEHA-BPNN in the case of 0dB and 6dB MSNR respectively. By observing Fig. 5 (a) that the modulation recognition accuracy of OQAM and QASK signals is low, these two signals are prone to confusion, and the recognition accuracy of the remaining signals is above $90 \%$. The main reason is that there are changes in the signal amplitude of these two kinds of signals, which leads to the high similarity of the extracted characteristic when the MSNR is low and making it hard to distinguish. By observing Fig. 5 (b) that when the MSNR is $6 \mathrm{~dB}$, there are still a small number of samples that can not be distinguished between OQAM and QASK, but the recognition accuracy of each signal has been greatly improved and all achieve above 95\%.

We compare the proposed QEHA-BPNN model with different existing methods, the conventional BPNN approach [19], the SVM approach [7], and the random forest (RF) approach [20]. These four modulation recognition techniques are applied to identify 8 modulation signals under the impulsive noise environment. Fig. 6 shows the overall recognition accuracy when the characteristics exponent $\alpha$ is 1.5 and MSNR varies from $-10 \mathrm{~dB}$ to $10 \mathrm{~dB}$. It can be seen that the overall recognition accuracy is improved with the 
increase of MSNR. It can be explained that the higher the MSNR, the closer the signal to the original appearance, making the extracted features easier to distinguish different modulation signals. Comparing the three traditional methods BPNN, SVM, and RF, the performance of SVM and RF is generally better than the conventional BPNN. Therefore, the QEHA is utilized to optimize the performance of the BPNN. It can be found that the recognition accuracy under the condition of MSNR $\leq 0 \mathrm{~dB}$ is significantly higher than other methods, and the recognition accuracy is above $99 \%$ when MSNR greater than $2 \mathrm{~dB}$.

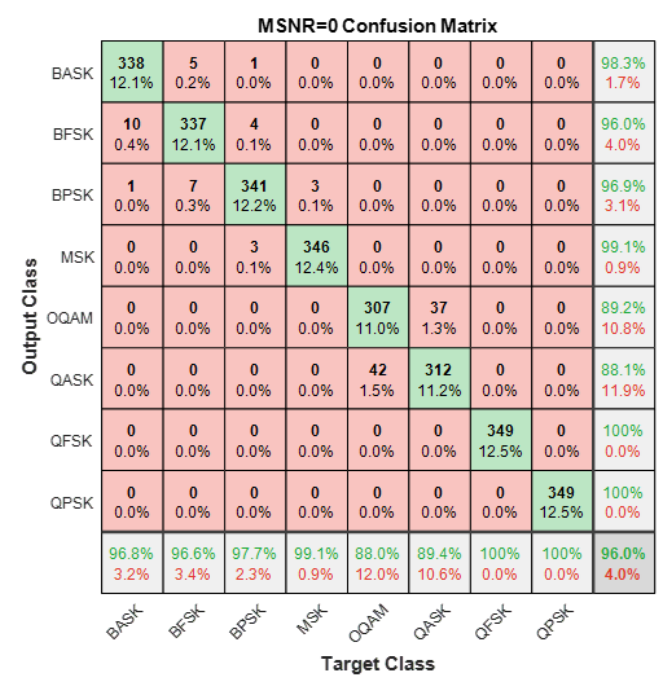

(a)

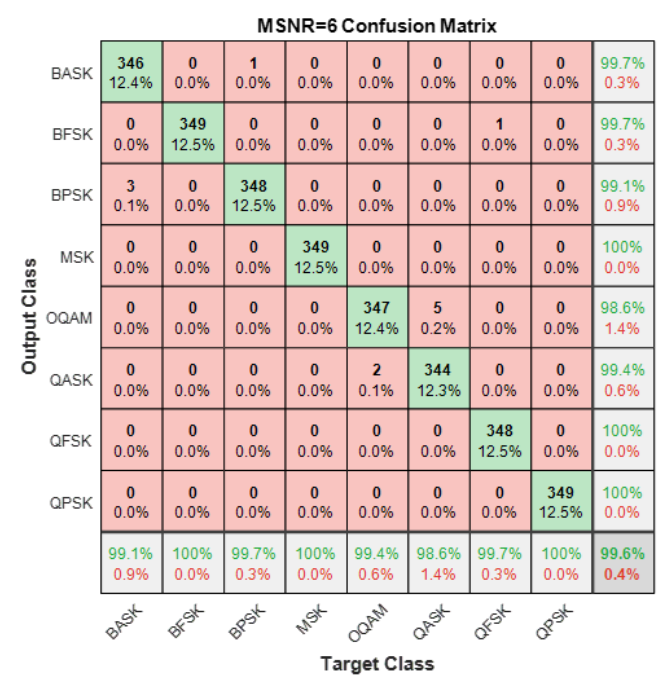

(b)

Fig. 5. QEHA-BPNN confusion matrix for the modulation recognition. (a) MSNR $=0 \mathrm{~dB}$ and (b) MSNR $=6 \mathrm{~dB}$.

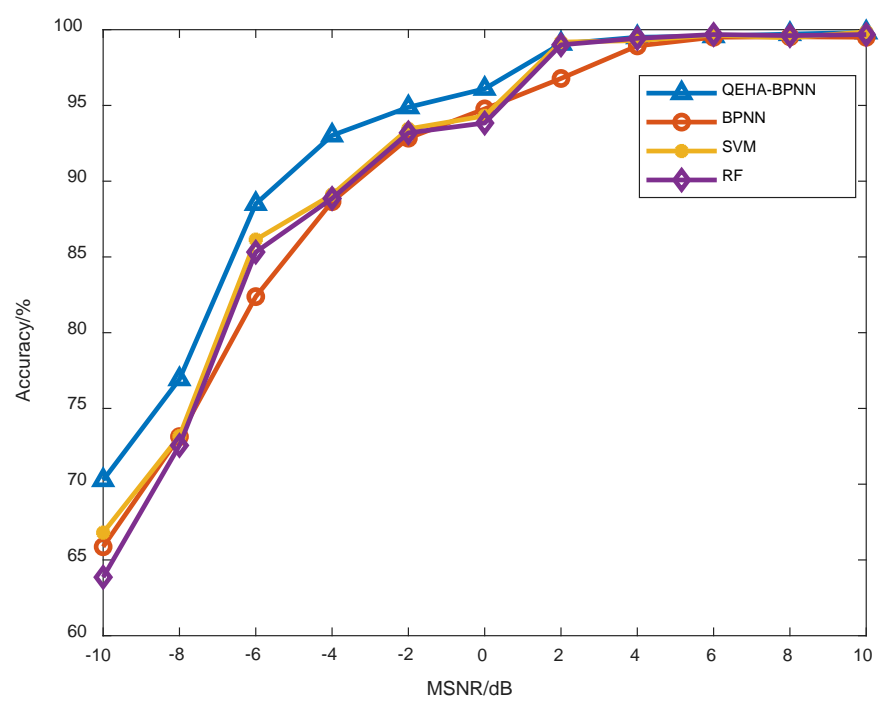

Fig. 6. Overall recognition accuracy of 4 recognition approach with $\alpha=1.5$ in different MSNR. 
Table 3 exhibits recognition accuracy for 8 modulation signals obtained by four methods respectively with characteristics exponent $\alpha$ is 1.5 and MSNR is $0 \mathrm{~dB}$. It is clearly seen from the table that the automatic modulation recognition accuracy of QASK and OQAM is lower than others, and the QFSK, QPSK almost achieve 100\%. The QEHA-BPNN method achieves better results in the identification of various modulation signals. The simulation results show that this scheme can automatically search the initial weights and thresholds of the BPNN, thereby effectively identifying eight modulation signals, which also illustrate the effectiveness of our proposed QEHA-BPNN method in impulsive noise environment.

Table 3. Recognition accuracy for 8 modulation signals with $\alpha=1.5$ and MSNR $=0 \mathrm{~dB}$.

\begin{tabular}{lllll}
\hline \multirow{2}{*}{ Modulation type } & \multicolumn{3}{c}{ Classifier } \\
\cline { 2 - 5 } & QEHA-BPNN & BPNN & SVM & RF \\
\hline BASK & $\mathbf{9 7 . 1 \%}$ & $95.7 \%$ & $93.1 \%$ & $94.8 \%$ \\
QASK & $\mathbf{8 8 . 8 \%}$ & $82.5 \%$ & $82.5 \%$ & $81.9 \%$ \\
BFSK & $\mathbf{9 6 . 3 \%}$ & $94.8 \%$ & $92.6 \%$ & $91.4 \%$ \\
QFSK & $\mathbf{1 0 0 \%}$ & $99.7 \%$ & $100 \%$ & $100 \%$ \\
BPSK & $\mathbf{9 8 . 9 \%}$ & $98.0 \%$ & $98.3 \%$ & $96.8 \%$ \\
QPSK & $\mathbf{1 0 0 \%}$ & $100 \%$ & $100 \%$ & $100 \%$ \\
MSK & $\mathbf{9 9 . 4 \%}$ & $99.1 \%$ & $99.4 \%$ & $99.4 \%$ \\
OQAM & $88.3 \%$ & $88.3 \%$ & $\mathbf{8 8 . 5 \%}$ & $86.2 \%$ \\
\hline
\end{tabular}

\subsection{Performance Comparisons of QEHA-BPNN with Other Optimal Algorithm}

We also use other optimization algorithms to optimize BPNN further verify the effectiveness of the proposed algorithm, including the PSO-BPNN approach [21], GA-BPNN approach [22], BAS-BPNN approach [23], and EHO-BPNN approach. Fig. 7 shows the overall recognition accuracy of 5 optimization schemes and conventional BP neural network for 8 modulation signals recognition with $\alpha=1.5$ and MSNR changes from $-10 \mathrm{~dB}$ to $10 \mathrm{~dB}$ and with $2 \mathrm{~dB}$ interval.

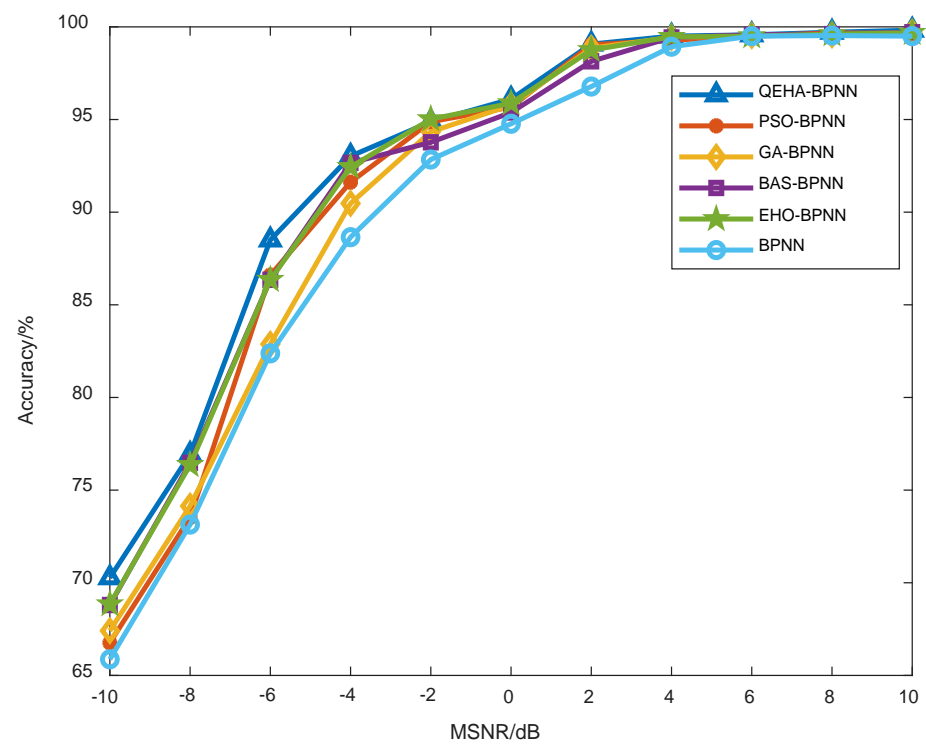

Fig. 7. Overall recognition accuracy of 5 optimization schemes and conventional BPNN with $\alpha=1.5$ in different MSNR. 
From Fig. 7 we can observe that the recognition accuracy of the optimized BPNN by optimization algorithms is higher than conventional BPNN, indicating that the performance of BPNN can be improved by using optimization algorithm. The overall recognition accuracy curves of several optimization schemes take on a similar trend. The accuracy of the QEHA-BPNN method is slightly higher than other optimization methods at low MSNR, and all of them can achieve above 99\% accuracy at high MSNR. The main reason is that these algorithms are based on the fitness value of the individual population to select the optimal value, which belongs to the global optimization method, thus reducing the possibility of the BPNN falling into a local minimum. However, the QEHA is improvement of the EHO algorithm, the biological mechanism is relatively simple, which makes it simpler to use and can achieve better results in most situations. Another advantage of QEHA is faster convergence speed, the simulation results are shown in Fig. 8.

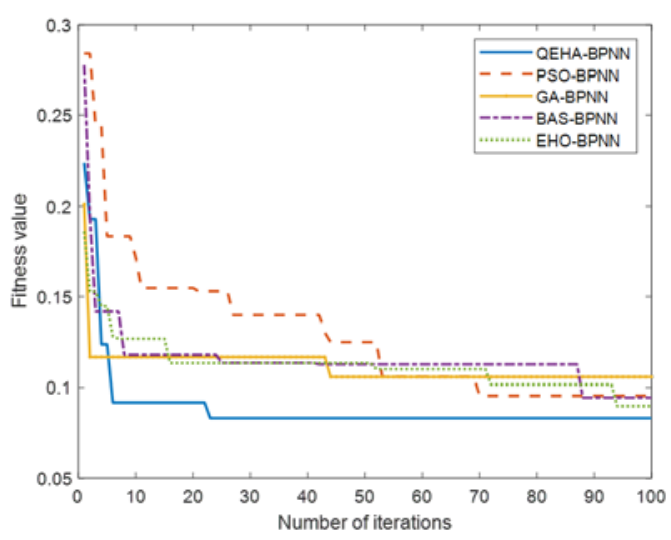

Fig. 8. Fitness value of 5 optimization algorithms with $\alpha=1.5$ and MSNR $=0 \mathrm{~dB}$ in different iterations.

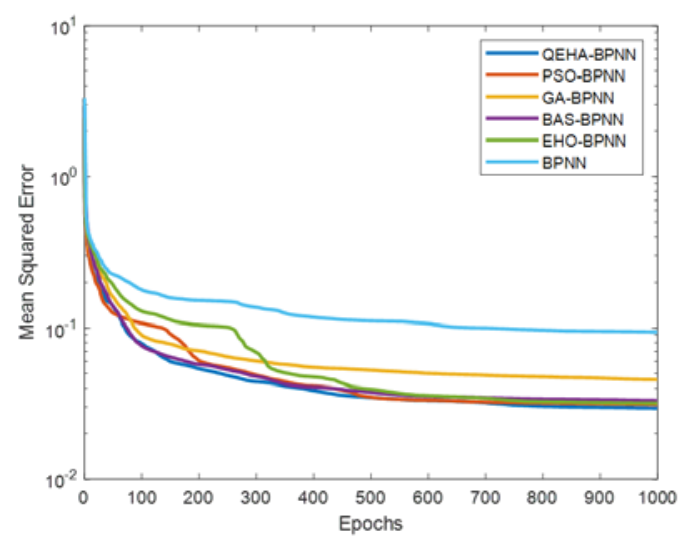

Fig. 9. The training convergence performance of 5 optimization schemes and conventional BPNN with $\alpha=1.5$ and MSNR = $0 \mathrm{~dB}$ in different epochs

Fig. 8 is a comparison curve of the optimal fitness value of each generation group of each algorithm under the condition that the characteristics exponent $\alpha$ is 1.5 and MSNR is 0dB in different iterations. It can be seen from the observation of Fig. 9 that the QEHA designed in this paper is significantly better than the other four algorithms in convergence speed and convergence accuracy is higher. Since the QEHA is based on the EHO algorithm and computing mechanism, uses quantum rotation gate and quantum not gate to evolve the quantum state of the quantum elephant. In the process of evolution, past historical information of individuals is effectively used, and the global convergence ability of the algorithm is enhanced.

Fig. 9 is the training convergence curve of 5 optimization schemes and the conventional BP neural network with characteristics exponent $\alpha$ is 1.5 and MSNR is 0dB in different training epochs. In order to observe the curve more clearly, this paper takes the logarithm of mean squared error (MSE). The training performance intuitively reflects the superior capability of the QEHA, indicating that the initial weights and thresholds of the BPNN searched by QEHA reduce the possibility that the BPNN is trapped in local minimum, accelerates the speed of global error reduction, and has good anti-noise performance. 


\section{Conclusion}

In this paper, we designed a novel low complexity algorithm QEHA based on EHO algorithm and quantum computing mechanism and proposed a modulation recognition method based on adaptive weighted myriad filter and QEHA-BPNN model in impulsive noise environment. The modulated signals passing through the impulsive noise channel are first preprocessed by an adaptive weighted myriad filter to suppress impulsive noise. Subsequently, instantaneous characteristics and high-order cumulants features are extracted as the classification feature set. Finally, BPNN optimized by QEHA is used as a classifier to recognition eight kinds of modulation signals effectively. The experimental results show that the adaptive weighted myriad filter has a good suppression effect on impulsive noise, which greatly improves the accuracy of modulation recognition in impulsive noise environment. And compared with other traditional pattern recognition classifiers, the proposed QEHA-BPNN classifier has significantly improved the modulation recognition accuracy under the condition of low MSNR. Moreover, the QEHA designed in this paper can enhance the global convergence ability of the EHO, compared with other classical optimization algorithms, QEHA has faster convergence speed and higher convergence accuracy, which shows that QEHA has better versatility and is easy to transplant to other engineering optimization problems.

The adaptive weighted myriad filter has the disadvantage of parameter estimation, so in future work, we will use an intelligent optimization algorithm to obtain the relevant parameters of the weighted myriad filter to reducing the dependence on prior knowledge.

\section{Acknowledgment}

This work was supported by the National Natural Science Foundation of China (No. 61571149), the Natural Science Foundation of Heilongjiang Province (No. LH2020F017), the Initiation Fund for Postdoctoral Research in Heilongjiang Province (No. LBH-Q19098), Heilongjiang Province Key Laboratory of High Accuracy Satellite Navigation and Marine Application Laboratory, and Key Laboratory of Advanced Marine Communication and Information Technology, Ministry of Industry and Information Technology.

\section{References}

[1] J. B. Tamakuwala, "New Low Complexity Variance Method for Automatic Modulation Classification and Comparison with Maximum Likelihood Method," in Proc. of 2019 International Conference on Range Technology (ICORT), Balasore, India, pp. 1-5, February. 2019. Article(CrossRefLink)

[2] J. Y. Chen, H. Cui, S. H. Miao and C. G. Wu, "FEM: Feature extraction and mapping for radio modulation classification," Physical Communication, vol 45, pp.1-8, April. 2021. Article(CrossRefLink)

[3] H. Han, Z. Y. Ren, L. Li and Z. G. Zhu, "Automatic Modulation Classification Based on Deep Feature Fusion for High Noise Level and Large Dynamic Input,” Sensor, vol.21, no.6, pp.1-13, March. 2021.

[4] W. Xie, S. Hu, C. Yu, P. Zhu, X. Peng and J. Ouyang, "Deep Learning in Digital Modulation Recognition Using High Order Cumulants,” IEEE Access, vol. 7, pp. 63760-63766, May. 2019. Article(CrossRefLink)

[5] C. Park, J. Choi, S. Nah, W. Jang and D. Y. Kim, “Automatic Modulation Recognition of Digital Signals using Wavelet Features and SVM,” in Proc. of 2008 10th International Conference on Advanced Communication Technology, Gangwon-Do, pp. 387-390, February. 2008. Article(CrossRefLink) 
[6] S. Taira and E. Murakami, "Automatic classification of analogue modulation signals by statistical parameters," in Proc. of MILCOM 1999. IEEE Military Communications. Conference Proceedings, Atlantic City, NJ, USA, pp. 202-207, November.1999. Article(CrossRefLink)

[7] S. Hassanpour, A. M. Pezeshk and F. Behnia, "Automatic Digital Modulation Recognition Based on Novel Features and Support Vector Machine,” in Proc. of 2016 12th International Conference on Signal-Image Technology \& Internet-Based Systems (SITIS), Naples, pp. 172-177, November. 2016. Article(CrossRefLink)

[8] Y. Ettefagh, M. H. Moghaddam and S. Eghbalian, “An adaptive neural network approach for automatic modulation recognition," in Proc. of 2017 51st Annual Conference on Information Sciences and Systems (CISS), Baltimore, MD, pp. 1-5, March. 2017. Article(CrossRefLink)

[9] X. Yan, G. Liu, H. Wu, G. Zhang, Q. Wang and Y. Wu, "Robust Modulation Classification Over $\alpha$-Stable Noise Using Graph-Based Fractional Lower-Order Cyclic Spectrum Analysis,” IEEE Transactions on Vehicular Technology, vol. 69, no. 3, pp. 2836-2849, January 2020. Article(CrossRefLink)

[10] T. V. R. O. Câmara, A. D. L. Lima, B. M. M. Lima, A. I. R. Fontes, A. D. M. Martins and L. F. Q. Silveira, "Automatic Modulation Classification Architectures Based on Cyclostationary Features in Impulsive Environments,” IEEE Access, vol. 7, pp. 138512-138527, September. 2019. Article(CrossRefLink)

[11] X. Tian, X. Sun, X. Yu and X. Li, "Modulation Pattern Recognition of Communication Signals Based on Fractional Low-Order Choi-Williams Distribution and Convolutional Neural Network in Impulsive Noise Environment," in Proc. of 2019 IEEE 19th International Conference on Communication Technology (ICCT), Xi'an, China, pp. 188-192, January. 2020. Article(CrossRefLink)

[12] C. C. Wang, M. Q. Liu, Q. Chen, B. D. Shang and H. Y. Tang, “Automatic digital modulation recognition in the presence of alpha-stable noise,” Physical Communication, vol. 43, pp. 1-8, October. 2020. Article(CrossRefLink)

[13] Sudhakar Kalluri and Gonzalo R. Arce, “Adaptive Weighted Myriad Filter Algorithms for Robust Signal Processing in $\alpha$-Stable Noise Environments,” IEEE Transactions on Signal Processing, vol. 46, no. 2, pp. 322-334, February.1998. Article(CrossRefLink)

[14] T. K. Roy, M. F. Pervej and M. Morshed, "Performance comparison of three optimized alternative pulse shaping filters with the raised cosine filter for wireless applications," in Proc. of 2015 International Conference on Computer and Information Engineering (ICCIE), Rajshahi, Bangladesh, pp. 9-12, February. 2015. Article(CrossRefLink)

[15] G. Wang, S. Deb and L. d. S. Coelho, "Elephant Herding Optimization," in Proc. of 2015 3rd International Symposium on Computational and Business Intelligence (ISCBI), Bali, Indonesia, pp. 1-5, January. 2015. Article(CrossRefLink)

[16] M. Nayak, S. Das, U. Bhanja and M. R. Senapati, "Elephant herding optimization technique based neural network for cancer prediction," Indormatics in Medicine Unlocked, vol. 21, October. 2020. Article(CrossRefLink)

[17] A. A. K. Ismaeel, I. A. Elshaarawy, E. H. Houssein, F. H. Ismail and A. E. Hassanien, "Enhanced Elephant Herding Optimization for Global Optimization," IEEE Access, vol. 7, pp. 34738-34752, March. 2019. Article(CrossRefLink)

[18] H. Gao and M. Diao, "Quantum Particle Swarm Optimization for MC-CDMA Multiuser Detection," in Proc. of 2009 International Conference on Artificial Intelligence and Computational Intelligence, Shanghai, China, pp. 132-136, January. 2009. Article(CrossRefLink)

[19] M. Yuan, L. Cheng, J. J. Dang and Y. Shi, "Recognition of Digital Modulation Signals Based on BP Neural Network,” Computer \& Digital Engineering, Vol. 47, No. 8, pp. 2075-2079, March. 2019. Article(CrossRefLink)

[20] Z. J. Tan, J. H. Shi, J. F. Hu, “ Low-Order Digital Modulation Recognition Algorithm based on Random Forest," Communication Technology, vol. 51, No. 3, pp. 527-532, March. 2018. Article(CrossRefLink) 
[21] W. Shi, D. Liu, X. Cheng, Y. Li and Y. Zhao, "Particle Swarm Optimization-Based Deep Neural Network for Digital Modulation Recognition,” IEEE Access, vol. 7, pp. 104591-104600, July. 2019. Article(CrossRefLink)

[22] X. Q. Wu, Y. Gao, "Novel Modulation Recognition Algorithm based on Genetic BP Neutral Network,” Communications Technology, vol.52, no.03, pp.529-534, March. 2019. Article(CrossRefLink)

[23] C. Zhang, S. Yu, G. Li and Y. Xu, "The Recognition Method of MQAM Signals Based on BP Neural Network and Bird Swarm Algorithm,” IEEE Access, vol. 9, pp. 36078-36086, February. 2021. Article(CrossRefLink)

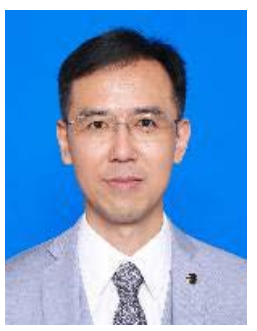

Hongyuan Gao received the Ph.D. degree from the Department of Communication and Information Systems, College of Information and Communication Engineering, Harbin Engineering University, China, in 2010. He has been a Visiting Research Professor under supervision of Prof. Minho Jo with the Department of Computer and Information Science, Korea University, Sejong Metropolitan City, South Korea, from 2015 to 2016 . He is currently an Associate Professor with the College of Information and Communication Engineering, Harbin Engineering University, China. Areas of his current interests include wireless energy harvesting communications, intelligent computing, artificial intelligence, radio signal recognition and classification, array signal processing, cognitive radio, HetNets in $5 \mathrm{G}$, communication theory, image processing, and massive MIMO.

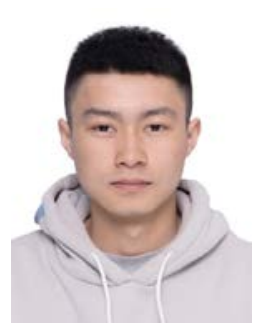

Shihao Wang received his B.E. degree in Information Countermeasure Technology from Harbin Engineering University, Harbin, Heilongjiang, P. R. China, in June 2019. He is currently a postgraduate student in Harbin Engineering University. His current research interests include intelligent computing, machine learning, modulation signal recognition and UAV cluster information interaction.

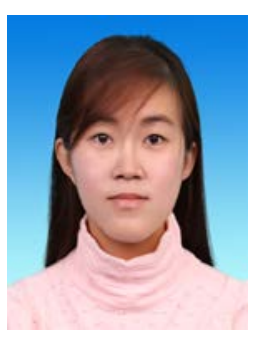

Yumeng Su received the B.Eng. degree in electronic information engineering from Harbin Engineering University, Harbin, Heilongjiang, China, in 2016. She is currently working toward the Ph.D. degree with Harbin Engineering University. Her current research interests include intelligent computing, resource management, secure communications, massive MIMO, co-frequency co-time full-duplex systems, network slicing, and beyond 5G technologies.

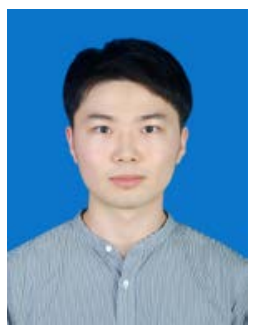

Helin Sun received his B.E. degree in Information and Communication Engineering from Harbin Engineering University, Harbin, Heilongjiang, P. R. China, in June 2020. He is currently a postgraduate student in Harbin Engineering University. His current research interests include intelligence computing and array signal processing.

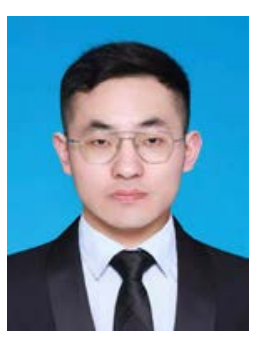

Zhiwei Zhang received his B.E. degree in Communication Engineering from Northeast Forestry University, Harbin, Heilongjiang, P. R. China, in June 2018. He is currently a postgraduate student in Harbin Engineering University. His current research interests include intelligent computing, blind source separation and array signal processing. 